\title{
Segregation of Behavior-Specific Synaptic Inputs to a Vertebrate Neuronal Oscillator
}

\author{
Jenifer Juranek ${ }^{1}$ and Walter Metzner ${ }^{2}$ \\ ${ }^{1}$ Program in Neuroscience, Department of Psychology and ${ }^{2}$ Department of Biology, University of California at Riverside, \\ Riverside, California 92521-0427
}

\begin{abstract}
Although essential for understanding the mechanisms underlying sensorimotor integration and motor control of behaviors, very little is known about the degree to which different behaviors share neural elements of the sensorimotor command chain by which they are controlled. Here, we provide, to our knowledge, the first direct physiological evidence that various modulatory premotor inputs to a vertebrate central pattern generator, the pacemaker nucleus in gymnotiform electric fish, carrying distinctly different behavioral information, can remain segregated from their various sites of origin in the diencephalon to the synaptic termination sites on different target neurons in the medullary pacemaker nucleus. During pharmacological activation of each of the premotor inputs originating from the three prepacemaker nuclei so far identified, we determined in vivo the changes in input resistance in the neuronal elements of the pacemaker nucleus, i.e., relay cells and pacemaker cells. We found that each input yields significantly different effects on
\end{abstract}

The wide range of behaviors seen in most animals often requires different activation patterns of the same motor output, i.e, the contraction of leg muscles during walking and swimming. An important question is, therefore, how much of the neural circuitry is uniquely used for the control of one particular behavior and how much of it is shared to control different motor functions. In rhythmic behaviors, switching between different motor patterns often involves changes in the modulatory input to the rhythmproducing central pattern generator (Harris-Warrick and Marder, 1991; Arshavsky et al., 1993; Katz, 1995; Marder and Calabrese, 1996). Relatively little, however, is known about whether multiple descending inputs to a single pattern-generating network act strictly independently or whether they also interact with one another (Metzner, 1993; Brodfuehrer and Burns, 1995; Heiligenberg et al., 1996; Blitz and Nusbaum, 1997).

The pacemaker nucleus of weakly electric gymnotiform fish represents such a neuronal network in which separate modulatory inputs generate distinct behavioral motor patterns by altering the discharge rate of its neuronal components (see Fig. 1) (Heiligenberg, 1991; Metzner and Viete, 1996a,b; Moortgat et al., 1998). For orientation and communication purposes, these fish produce

\footnotetext{
Received June 9, 1998; revised Aug. 4, 1998; accepted Aug. 11, 1998.

This work was supported by academic research grants from University of California at Riverside and by the National Science Foundation. We thank C. Condon, S. Currie, K. Moortgat, G. Stanley, and S. Viete for their comments on this study. Y. T. Yan, D. Welsbie, Y. Song, and B. Andersen provided assistance with histological procedures and data analysis.

Correspondence should be addressed to Walter Metzner, Department of Biology, University of California at Riverside, Riverside, CA 92521-0427.

Copyright (C) 1998 Society for Neuroscience $\quad 0270-6474 / 98 / 189010-10 \$ 05.00 / 0$
}

these cells; the inputs from the two diencephalic prepacemaker nuclei, PPnC and PPnG, which resulted in increased oscillator activity, caused significantly lower input resistances in relay and pacemaker cells, respectively, exhibiting drastically different time courses. The input from the sublemniscal prepacemaker nucleus, which resulted in reduced oscillator activity, however, caused a significant increase in input resistance only in relay cells. Considering that the sensory pathways processing stimuli yielding these behaviors are separated as well, this study indicates that sensorimotor control of different behaviors can occur in strictly segregated channels from the sensory input of the brain all through to the synaptic input level of the final premotor command nucleus.

Key words: premotor control; parallel pathways; input resistance; Eigenmannia; pacemaker nucleus; jamming avoidance response; communication behavior

electric signals by discharging an electric organ situated in the tail region. Each electric organ discharge (EOD) is triggered by a medullary pacemaker nucleus that is composed of two types of neurons, pacemaker and relay cells. Relay cells project to spinal motoneurons that innervate the electrocytes of the electric organ. Pacemaker cells are connected with each other and with relay cells via mixed chemical and electrical synapses (Bennett, 1971; Elekes and Szabo, 1982, 1985).

In the electric knife fish Eigenmannia, three afferent synaptic inputs to the pacemaker nucleus are known so far (see Fig. 1). All inputs and intrinsic connections are excitatory (Kennedy and Heiligenberg, 1994) and presumably use Glu as their natural transmitter. One input generates chirp-like communicatory signals. It arises from the ventrolateral portion of a diencephalic prepacemaker nucleus (PPnC) and is mediated by non-NMDAtype receptors (Kawasaki et al., 1988; Dye et al., 1989; Metzner, 1993; Juranek and Metzner, 1997). The second input is part of a pathway that generates increases in EOD frequency that occur during a particular behavior related to orientation and prey detection, the jamming avoidance response (JAR) (Bullock et al., 1972). It originates from the dorsal portion of the diencephalic nucleus electrosensorius (Keller and Heiligenberg, 1989; Keller et al., 1990), sending excitatory connections to the medial portion of the diencephalic prepacemaker $(\mathrm{PPnG})$, which projects to the pacemaker nucleus possibly via both NMDA and non-NMDAtype receptors (Metzner, 1993; Spiro et al., 1994; Juranek and Metzner, 1997). The third pathway controls frequency decreases during the JAR and originates in the ventral nucleus electrosensorius (nE $\downarrow$ ) (Keller and Heiligenberg, 1989; Keller et al., 1990). 
It provides GABAergic input to the mesencephalic sublemniscal prepacemaker nucleus (SPPn). The SPPn is tonically active and also controls the EOD frequency, even in the absence of jamming signals. Its projection to the pacemaker nucleus is mediated by NMDA-type receptors (Metzner, 1993; Spiro et al., 1994; Juranek and Metzner, 1997).

Here, we provide direct physiological evidence indicating that the segregation of these separate, behaviorally distinct premotor pathways extends even to the level of their synaptic targets in the pacemaker nucleus. Inputs yielding behaviors that involve increases in oscillatory firing rate, such as chirping and EOD accelerations during the JAR, affect different target cells, relay and pacemaker cells, respectively. In contrast, the inputs mediating chirping and EOD decelerations during the JAR, respectively, both affect relay cells, but yield opposite physiological effects, consistent with the involvement of different receptor subtypes.

\section{MATERIALS AND METHODS}

Nineteen Eigenmannia (12-15 cm body length) were used for this study. The animals were obtained from a tropical fish wholesaler (Bailey's, San Diego, CA). The surgical techniques follow earlier studies (Dye et al., 1989; Kawasaki and Heiligenberg, 1989; Metzner, 1993; Juranek and Metzner, 1997). They are in accordance with National Institutes of Health guidelines for experiments involving vertebrate animals and were approved by the local Animal Care Committee. Briefly, fish were immobilized by injecting a $2 \%$ flaxedil solution (gallamine triethiodide; $2-4 \mu \mathrm{l}$, i.m.; Sigma, St. Louis, MO) and gently suspended in the center of an aquarium (resistivity, $9-12 \mathrm{k} \Omega \mathrm{cm} ; 26-28^{\circ} \mathrm{C}$ ) by a pair of foam-lined forceps. Although Flaxedil strongly attenuated the EODs of Eigenmannia, residual signals (50 $\mu \mathrm{V}$ to $1 \mathrm{mV}$ ), locked to the spinal command cells, could still be monitored with a suction electrode fitted over the tip of the tail. Only the dorsal surface of the fish's head was exposed above the water surface. The fish was respirated with a stream of aquarium water (3-4 drops/sec) via a glass tube inserted inside its mouth. After application of $2 \%$ lidocaine (Western Medical Supplies, Arcadia, CA), a small hole ( $<1 \mathrm{~mm}$ in diameter) was made in the occipital bone. The medullary pacemaker nucleus was then located $\sim 2500-3000$ $\mu \mathrm{m}$ below the cerebellar surface (see below). An additional hole was made by removing parts of the parietal bone unilaterally to allow for penetration of the diencephalic prepacemaker nuclei (PPnC and PPnG) and the $\mathrm{nE} \downarrow, \sim 1500-2100 \mu \mathrm{m}$ below the tectal surface. To stabilize the fish, a small plexiglass rod held by a micromanipulator was glued to a small area of the remaining contralateral parietal bone.

We selectively elicited the characteristic EOD frequency modulations occurring during chirping behavior and the JAR while we recorded intracellular changes of membrane potential in the two cell types of the pacemaker nucleus. By means of two separate microdrives (Burleigh Inchworm, Fishers, NY, and Narishige, Tokyo, Japan), one microelectrode was placed in the pacemaker nucleus for intracellular recordings and another electrode (tampered tip with 5-10 $\mu \mathrm{m}$ diameter) was advanced into one of the premotor nuclei, PPnC, PPnG, or $\mathrm{nE} \downarrow$, to pharmacologically activate its neurons (iontophoretic injection of $0.1 \mathrm{M} \mathrm{L}-\mathrm{Glu}, \mathrm{pH} 8$, $\sim 100 \mathrm{nA}$ negative DC). In Eigenmannia, it is quite difficult to localize and sufficiently inhibit the sublemniscal prepacemaker to generate a consistent drop in EOD frequency. Therefore, we used stimulation of the $\mathrm{nE} \downarrow$ (see Fig. 1) with L-Glu. The nE $\downarrow$ provides GABAergic input to the SPPn, and thus its stimulation causes a decrease in EOD frequency (Keller et al., 1990; Metzner, 1993; Juranek and Metzner, 1997).

Before intracellular recordings from pacemaker and relay cells could be performed, the exact location of the unpaired pacemaker nucleus had to be determined by using a glass capillary filled with $3 \mathrm{M} \mathrm{NaCl}$ for recordings of its field potential. After localization of the pacemaker nucleus, the NaCl-filled capillary was removed, and a glass micropipette filled with $2 \%$ neurobiotin (Vector Laboratories, Burlingame, CA) in $3 \mathrm{M} \mathrm{KCl}$ (borosilicate glass pulled on a Sutter P-87; 30-50 M $\Omega$ ) was advanced to the same location for intracellular recordings. Penetrations of pacemaker and relay cells were achieved by applying brief overcompensation of capacitance neutralization and/or slight mechanical tapping of the head stage of the microdrive. Once the membrane potential of each penetrated cell had stabilized and a steady baseline membrane potential was recorded, cells were characterized physiologically as either pacemaker or relay cells based on action potential wave forms; action potentials in pacemaker cells exhibited gradually depolarizing potentials between spikes that are lacking in relay cells (Bennett et al., 1967; Juranek and Metzner, 1997). After data collection from each cell, a positively biased sinusoidal current ( $\sim 2.0 \mathrm{nA}$ for $5-10 \mathrm{~min})$ was used to inject neurobiotin into the cell. Subsequent histological verification allowed us to unequivocally classify the cells as relay cells or pacemaker cells.

A high-impedance amplifier with active bridge circuitry (Intra 767; World Precision Instruments, New Haven, CT) was used to record the membrane potential of penetrated cells and to inject 0.5-msec-long hyperpolarizing constant current pulses ranging from $-2 \mathrm{nA}$ to $-6.5 \mathrm{nA}$. The fish's EOD was used to trigger the intracellular current pulses and to deliver them at a constant phase between each recorded spike during synaptically elicited frequency changes. The resolution of our input resistance measurements was $30 \mathrm{k} \Omega(0.2 \mathrm{mV})$. Intracellular signals and the fish's EODs were stored on video tape using a recording adapter (Vetter 3000A; sample rate, 40 and $20 \mathrm{kHz}$, respectively).

At the conclusion of each experiment, fish were killed in 3-aminobenzoic acid ethylester (MS-222; Sigma, St. Louis, MO) and transcardially perfused with $0.9 \%$ saline, followed by $4 \%$ paraformaldehyde in $0.1 \mathrm{M}$ phosphate buffer, $\mathrm{pH} 7.4$ (30 min). Subsequently, brains were removed and stored overnight in the fixative solution. The hindbrain was then coronally sectioned on a vibratome at $50 \mu \mathrm{m}$ intervals and collected in $0.02 \mathrm{M}$ PBS, pH 7.3. After a standard Vectastain ABC (Vector Laboratories) and DAB (Sigma) reaction (Heiligenberg et al., 1996; Metzner and Juranek, 1997a), the tissue was mounted onto subbed slides and allowed to dry before it was counterstained with neutral red and coverslipped with Permount (Fisher Scientific, Fair Lawn, NJ). Lightmicroscopical inspection allowed us to identify neurobiotin-filled cells. This provided histological verification of the physiological classification of cell types made during the actual experiment. Furthermore, the site of electrode impalement during intracellular recordings was indicated in our neurobiotin-filled cells by a slight rupture in the cell membrane. We only included recordings obtained from somata in our data sample.

For off-line data analysis, both EODs and intracellular recordings were analog-to-digital converted using a commercial data analysis system (sample rate, $40 \mathrm{kHz} /$ channel; Datawave, Denver, $\mathrm{CO})$. Spike rates were determined by zero-crossing analysis. Changes in input resistance were calculated by measuring the amplitude of downward voltage deflections produced by constant current pulses and dividing by the amount of current injected. 
Data obtained during PPnG and $\mathrm{nE} \downarrow$ stimulation are presented as mean \pm SEM of 10 consecutive measurements. Data obtained during chirping elicited during PPnC stimulation were not averaged and are presented in their raw format, because the rapid changes during chirps occur over the course of only a few EOD cycles and each chirp usually covers a different EOD frequency change. All changes in input resistance were normalized to the average input resistance of each cell before pharmacological manipulation of the various descending synaptic inputs. Changes in input resistance were plotted as a function of frequency changes using linear regression techniques (SigmaPlot; Jandel Scientific, San Rafael, CA). All but one (effect of nE $\downarrow$ stimulation on pacemaker cells) data set were normally distributed and varied equally with respect to the regression line. An analysis of covariance revealed that regression slopes for a given cell type were not significantly different $(p>0.25)$ for a common stimulation site. Therefore, data from each cell type were separately pooled, and a new regression line was calculated according to the method of least squares for each of these pooled data sets. Quantitative comparisons between cell types was done by comparing the slopes of each regression line and noting differences at the 0.001 level of significance.

\section{RESULTS}

The pacemaker nucleus in gymnotiform fish is composed of two morphologically distinct cell types: pacemaker cells and relay cells. In Eigenmannia, pacemaker cells are coupled both with each other and with relay cells via electrotonic and chemical synapses (Fig. 1) (Elekes and Szabo, 1982). The mixed chemical and electrotonic coupling between cells in the pacemaker nucleus poses a technical challenge for the identification of cellular effects occurring in response to changes in the activity of its synaptic inputs in vivo. Even exceptionally large hyperpolarizing currents injected intracellularly (Dye, 1991) were ineffective in halting oscillatory changes in membrane potential. Intracellular application of a voltage-gated sodium channel blocker QX-314 is also only partially effective and reduces the spike amplitudes of pacemaker and relay cells only by up to $50 \%$ (our unpublished observations). Therefore, measuring changes in input resistance appears to be the only direct means of quantifying postsynaptic effects of the inputs to the pacemaker nucleus in vivo.

Whereas the diameter of relay cells varies between 65 and 70 $\mu \mathrm{m}$, pacemaker cells are approximately half that size, with diameters ranging between 30 and $35 \mu \mathrm{m}$. Correspondingly, the input resistance of pacemaker cells was significantly different from that of relay cells (Mann-Whitney $U$ test; $p<0.001$ ). The smaller pacemaker cell type had an average input resistance of $3.7 \pm 0.30$ $\mathrm{M} \Omega(n=10)$, whereas the larger relay cell type showed an average input resistance of $2.7 \pm 0.11 \mathrm{M} \Omega(n=8)$. This overall range of input resistance in pacemaker and relay cells is consistent with earlier reports in other gymnotiform fish (Bennett et al., 1967; Spiro, 1997).

To assess whether voltage-gated conductance changes could have masked the effects caused by the synaptic input to pacemaker and relay cells, we determined the change in membrane voltage as a function of the amount of injected current (Fig. $2 A, B)$. A highly significant linear relationship $\left(r^{2}=0.99 ; p<\right.$ 0.001 ) was observed for changes in membrane potential caused by DC current injections. Hence, the input resistance, extracted from the slope of the regression lines, was independent of the amount of current injected and corresponded well to the average values given above [2.97 $\mathrm{M} \Omega$ for a relay cell (Fig. $2 A$ ) and 3.75

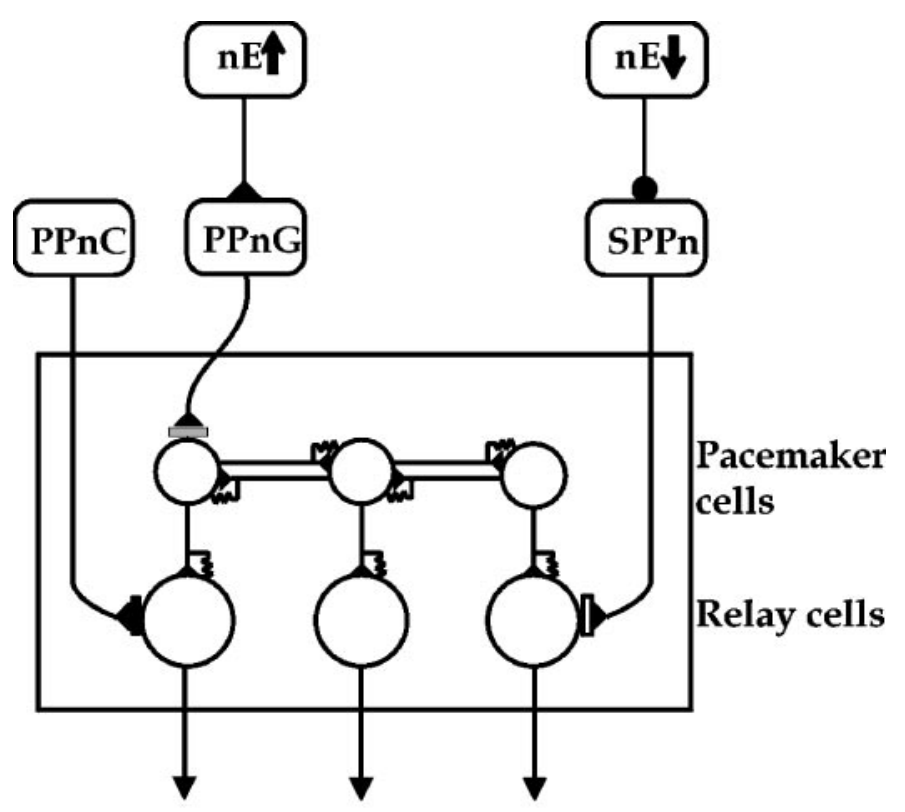

to spinal motor neurons of electric organ

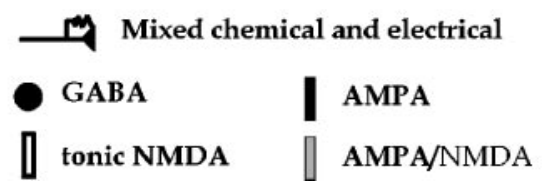

Figure 1. Schematic representation of the premotor circuitry in Eigenmannia based on this and previous studies involving pharmacological, anatomical, and histochemical techniques. Three separate glutamatergic inputs to the pacemaker nucleus control three different behavioral patterns: (1) chirp-like communication behavior, (2) EOD accelerations during JAR, and (3) EOD decelerations during JAR, as well as the resting EOD rate when no jamming signal is present. The present study provides direct physiological evidence for the termination of the three inputs onto different cell types, which was previously only based on preliminary indirect evidence (Kawasaki et al., 1988; Dye et al., 1989; Keller et al., 1990; Metzner, 1993; Heiligenberg et al., 1996; Juranek and Metzner, 1997; present study). $n E \uparrow$, Diencephalic nucleus electrosensorius.

$\mathrm{M} \Omega$ for a pacemaker cell (Fig. 2B)]. In addition, in both cell types, the height of the hyperpolarizing current pulses were unaffected by shifts in the membrane potential (Fig. $2 C, D$ ) over a behaviorally relevant frequency range (Fig. $2 E, F)$. Therefore, the changes in input resistance that we observed during activation of the three synaptic inputs to the pacemaker nucleus were not attributable to activation of voltage-gated ion channels intrinsic to pacemaker and relay cells but were, instead, only attributable to modulatory effects of the synaptic inputs.

We also tested whether input resistances of pacemaker cells and relay cells were correlated with different EOD resting frequencies in different fish ranging from 380 to $500 \mathrm{~Hz}$. No significant correlation between EOD resting frequency and input resistance in either of the two cell types was found (Spearman's rank test; $p>0.10)$.

In the following, we present physiological evidence for the assumption that the synaptic input from each of the three prepacemaker nuclei affects pacemaker and relay cells in a distinctly different manner. We measured the changes in input resistance in pacemaker cells and in relay cells during pharmacological activation or inhibition of the three premotor pathways terminating on 

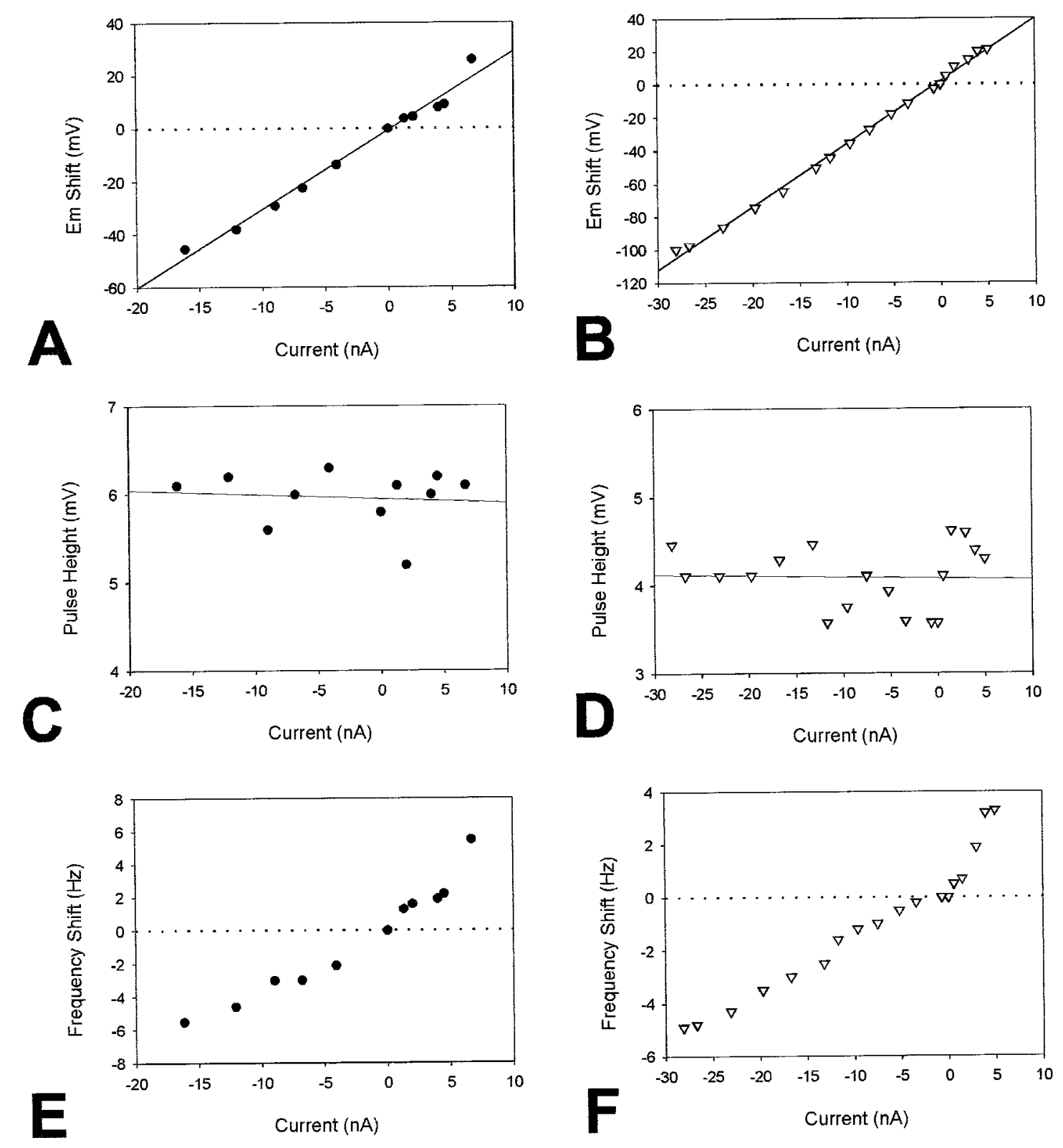

Figure 2. Changes in membrane potential attributable to injected DC current steps in a relay cell soma $(A)$ and a pacemaker cell soma $(B)$. The zero line in each plot represents a resting $E_{\mathrm{m}}$ of $-60 \mathrm{mV}$ and $-55 \mathrm{mV}$, respectively. A linear relationship $\left(r^{2}=0.99\right)$ is observed between injected current and shifts of membrane potential in each cell type, indicating that input resistance (represented by the slope of the regression line) did not significantly change with the amount of injected DC current. For the relay cell, the input resistance derived from the slope of the regression line was $2.97 \mathrm{M} \Omega$, and for the pacemaker cell, it was $3.75 \mathrm{M} \Omega$. C, $D$, Height of hyperpolarizing current pulses as a function of shifts in membrane potential caused by injection of DC current. Pulse height was unaffected by shifts of the membrane potential over a range even wider than that observed during the modulation of the synaptic inputs to the pacemaker nucleus. $E, F$, Injected current caused changes in spike frequency (and EOD; data not shown) that were behaviorally relevant.

these two cell types of the pacemaker nucleus (Fig. 1). Because it is difficult to localize and sufficiently inhibit the SPPn to generate a consistent drop in EOD frequency, we stimulated the $\mathrm{nE} \downarrow$ with $\mathrm{L}-\mathrm{Glu}$ instead. The $\mathrm{nE} \downarrow$ provides GABAergic input to the SPPn, and thus its stimulation causes a decrease in EOD frequency comparable with that caused by direct inhibition of the SPPn with GABA (Keller et al., 1990; Metzner, 1993; Juranek and Metzner, 1997).

We collected data from 12 pacemaker cell somata and 12 relay cell somata in 19 animals. During recordings from a particular cell type, each premotor input was repeatedly activated, or in the case of the $n E \downarrow-S P P n$, inhibited 2-15 times.

\section{Changes in input resistance during PPnC stimulation}

PPnC stimulation elicited brief accelerations in spike rate in both pacemaker cells and relay cells that lasted between 5 and $20 \mathrm{msec}$. The resulting modulations in spike rate varied between 30 and $160 \mathrm{~Hz}$ ( $n=31$ chirps in four relay cell somata; $n=36$ chirps in four pacemaker cell somata). Figure 3 exemplifies that, during similar frequency changes of $\sim 60 \mathrm{~Hz}$, the amplitude of the brief 


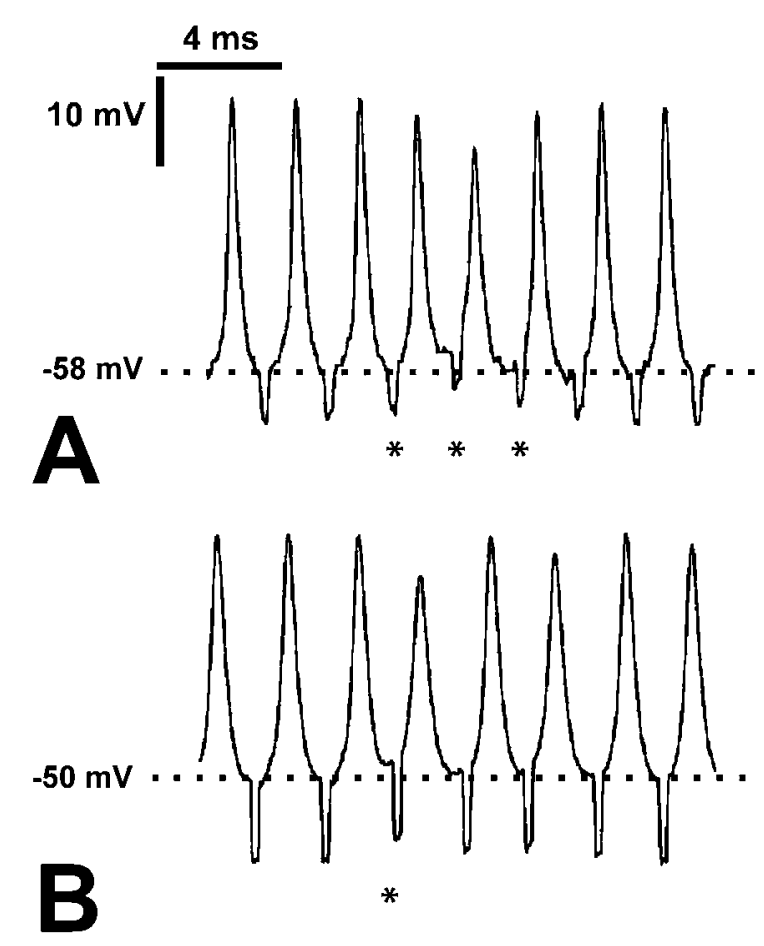

Figure 3. Representative input resistance changes measured in a relay cell soma $(A)$ and a pacemaker cell soma $(B)$ during a single chirp elicited by PPnC stimulation. Frequency-time plots of the same traces are given in Figure 4. Asterisks indicate spike intervals occurring during chirps. Downward voltage deflections were caused by $0.5 \mathrm{msec}$ hyperpolarizing constant current pulses, which were $2(A)$ and $3 \mathrm{nA}(B)$, respectively. During the chirp, the amplitude of the test pulses was reduced more profoundly in a relay cell $(A)$ than in a pacemaker cell $(B)$.

negative deflections in membrane voltage caused by hyperpolarizing current pulses was consistently reduced more profoundly in relay cells than in pacemaker cells. When quantified, the same recordings shown in Figure 3 reveal a 34\% decrease in the input resistance of the relay cell (Fig. $4 A$ ), whereas that of a pacemaker cell decreased only by $9 \%$ (Fig. $4 B$ ). These decreases in input resistance also showed different time courses in pacemaker and relay cells (Mann-Whitney $U$ test; $p<0.001$ ). In all relay cells tested, input resistances were reduced over an average duration of $13.02 \mathrm{msec}$ ( $\mathrm{SD} \pm 2.65 \mathrm{msec}$; range, 8.29-20.14 msec), whereas the average duration of decreased input resistances in pacemaker cells was only $9.88 \mathrm{msec}(\mathrm{SD} \pm 2.24 \mathrm{msec}$; range, 5.02-15.58 msec).

In summary (Fig. 4C), a linear regression analysis revealed for relay cells a significantly greater change in input resistance than in pacemaker cells $(p<0.001)$. In all relay cells analyzed, the input resistance decreased by $14.0 \mathrm{k} \Omega / \mathrm{Hz}\left(r^{2}=0.50\right)$, whereas in our entire sample of pacemaker cells, the input resistance decreased by merely $3.5 \mathrm{k} \Omega / \mathrm{Hz}\left(r^{2}=0.47\right)$. This decrease in input resistance observed in relay cells during $\mathrm{PPnC}$ stimulation is indicative of a large conductance increase characterized by a brief time course lasting between 8 and $20 \mathrm{msec}$.

Occasionally, brief interruptions of the ongoing spike rate did occur in relay cells, particularly after a dramatic acceleration in instantaneous spike frequency (EOD frequency followed that of the relay cell spikes) (Fig. 5). In all four relay cells analyzed, a skipped spike during an interruption was preceded by a "shoulder" on the repolarizing flank of the previous action potential. This shoulder consistently appeared at membrane potentials that were 10-25 $\mathrm{mV}$ depolarized relative to baseline (mean, $17.7 \mathrm{mV}$; $\mathrm{SD}=3.9 \mathrm{mV} ; n=54)$. After the skipped spike, instantaneous spike frequency was dramatically reduced yet recovered to the resting frequency in the next cycle. This phenomenon was never observed in pacemaker cell recordings. Furthermore, such shoulders never occurred during the rising phase of relay cell spikes.

Changes in input resistance during PPnG stimulation

Pharmacological stimulation of the PPnG with [scap]l-Glu elicited gradual frequency accelerations varying between 5 and 30 $\mathrm{Hz}$. We recorded from four pacemaker cell somata during seven stimulations of the PPnG and from four relay cell somata during eight PPnG stimulations. In contrast to PPnC stimulations, we observed a greater decrease in input resistance in pacemaker cells than in relay cells (Fig. 6A,B). In addition, the time courses of the decrease in input resistance were much slower during PPnG stimulation and reflected the slower and longer lasting frequency acceleration extending over a period of several seconds compared with the brief acceleration observed during stimulation of the PPnC, which lasted for only a few milliseconds. This difference is likely to be based on the involvement of different Glu receptor subtypes (NMDA and AMPA, respectively) and will be addressed in the Discussion.

Figure $6 C$ summarizes the changes in input resistance with changing frequency for our entire data pool. Input resistance in pacemaker cells decreased by $27.1 \mathrm{k} \Omega / \mathrm{Hz}\left(r^{2}=0.67\right)$, whereas in relay cells the input resistance was reduced by only $8.6 \mathrm{k} \Omega / \mathrm{Hz}$ $\left(r^{2}=0.66\right)$. The regression coefficients for the two regression lines were significantly different $(p<0.001)$.

\section{Changes in input resistance during SPPn inhibition}

Gradual frequency decelerations varying between 3 and $5 \mathrm{~Hz}$ and similar to those obtained by inhibiting the SPPn with GABA (Metzner, 1993) were elicited by stimulation of the $\mathrm{nE} \downarrow$ with L-Glu (see Materials and Methods, experimental procedures; Fig. 1 ). We tested the effects of nine stimulations of the $n E \downarrow$ in four relay cell somata and of eight $\mathrm{nE} \downarrow$ stimulations in four pacemaker cell somata. In contrast to decreases in input resistance observed during frequency accelerations attributable to either PPnC or PPnG stimulation, indirect SPPn inhibition resulted in increases in input resistance, whereas the spike (and EOD) frequency gradually decelerated. When quantified, during a $4 \mathrm{~Hz}$ frequency shift, the input resistance of a relay cell increased by $8 \%$ (Fig. $7 A$ ), whereas in the pacemaker cell, it increased by $5 \%$ (Fig. $7 B$ ).

During indirect inhibition of the SPPn, therefore, the input resistances in relay cells changed in a manner different from those observed in pacemaker cells (Fig. $7 C$ ). In relay cells, the input resistance increased by $42.8 \mathrm{k} \Omega / \mathrm{Hz}\left(r^{2}=0.72\right)$. In pacemaker cells, on the other hand, the change in input resistance was not linearly related to the decreases in spike (and EOD; data not shown) frequency $\left(r^{2}=0.07\right)$. Using nonlinear regression techniques, we tried to quantitatively describe the changes observed in pacemaker cells during EOD decelerations. However, the highly variable nature of these data points did not lend them to this form of analysis. Hence, we concluded that the responses of pacemaker cells were qualitatively different from the responses of relay cells, and only in relay cells, spike frequency (and EOD frequency) was clearly related with a change in input resistance. The increases in input resistance we observed in relay cells during SPPn inhibition is the first direct physiological evidence for EOD decelerations being mediated by a reduced level of tonic excitatory input. 

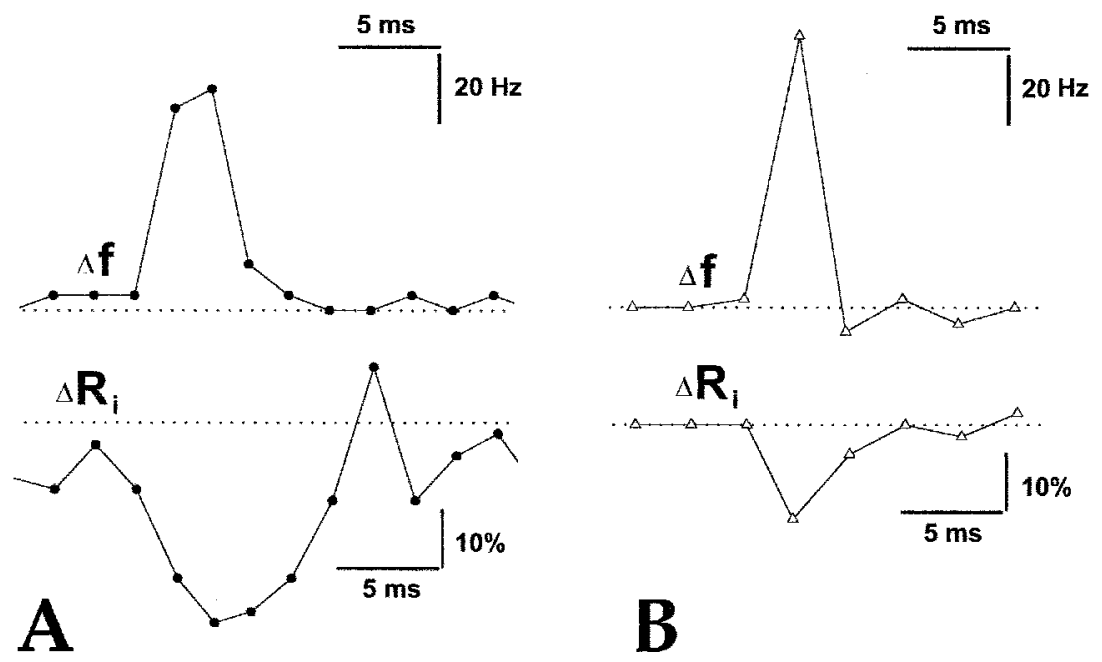

B
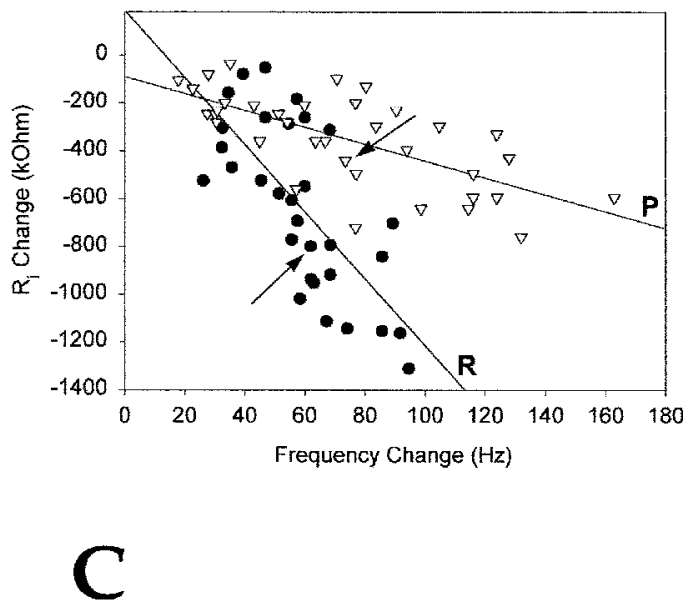

Figure 4. Time courses of changes in spike frequency $(\Delta f$, top traces $)$ and input resistance $\left(\Delta R_{\mathrm{i}}\right.$, bottom traces $)$ in a representative example of a recording from a relay cell $(A)$ and a pacemaker cell $(B)$ during the same chirps presented in Figure 3 . During the chirp, input resistances decreased significantly more in the relay cell than in the pacemaker cell (Mann-Whitney $U$ test; $p<0.001$ ). Resting frequencies (i.e., frequency before onset of chirp) were $488(A)$ and $362 \mathrm{~Hz}(B)$. Input resistances before frequency acceleration were $2.8(A)$ and $4.1 \mathrm{M} \Omega(B)$. $C$, Changes in input resistance as a function of maximum frequency changes during chirps for entire data sample of relay cells ( filled circles; 31 chirps in four somata) and pacemaker cells (open triangles; 36 chirps in four somata). The slope of each regression line was $-14.0 \mathrm{k} \Omega / \mathrm{Hz}$ for relay cells $(R)$ and $-3.5 \mathrm{k} \Omega / \mathrm{Hz}$ for pacemaker cells $(P)$. The regression coefficients of these two lines were significantly different $(p<0.001)$. Arrows indicate the examples depicted in Figures 3 and $4 A$, $B$.

In conclusion, as summarized in Figure 8, two forms of EOD frequency accelerations, rapid and gradual, are mediated by selective termination of glutamatergic inputs onto different cell types, relay cells and pacemaker cells, respectively. Quantitative differences in changes in input resistance between cell types for a given premotor input, e.g., from PPnC, is indicative of selective synapse formation; the greatest changes are expected to be observed in the target cell type. In contrast, EOD decelerations are attributable to "turning off" a tonic glutamatergic input to relay cells. Thus, opposing gradual frequency changes, decelerations and accelerations are independently controlled at the level of a single cell type, i.e., relay cells, by decreasing a tonic conductance and transiently increasing a conductance, respectively.

\section{DISCUSSION}

The three synaptic inputs to the pacemaker nucleus in gymnotiform electric fish are not only behaviorally strictly segregated (Kawasaki et al., 1988; Dye et al., 1989; Metzner, 1993; recent review: Metzner and Viete, 1996a,b) but, as we have shown here, also involve qualitatively and quantitatively different cell responses, as well as different target cells. This enables us to finally adopt a model circuit diagram (Fig. 1) that has been proposed previously (Metzner, 1993; Juranek and Metzner, 1997) but in which the innervation pattern of the cellular components of the pacemaker nucleus was only based on indirect evidence from pharmacological and preliminary physiological and histological experiments. This model (Fig. 1) accounts for the emergence of three different behavioral patterns from a single oscillatory network consisting of only two different cell types and the single neurotransmitter Glu. The observed small changes in input resistance seen also in "coupled cells", i.e., those cell types that do not represent primary targets for projections from the three premotor nuclei, cannot be explained by a presence of voltage-gated channels (Fig. 2). Although we cannot completely rule out marginal input from the three premotor structures to coupled cells, the effect could be attributable to the presence of intrinsic chemical synapses between pacemaker and relay cells (Bennett, 1971;
Elekes and Szabo, 1982, 1985) (Fig. 1). It could also be attributable to voltage-dependent changes in the conductance of the gap junctions between pacemaker and relay cells (for review, see Bennett et al., 1990; Bennett, 1997).

We are not aware of any other vertebrate system in which different premotor pathways mediating different behavioral patterns remain segregated to the level of the synaptic input to their target cells in the premotor command nucleus. In two of the best studied systems, the central pattern generators in the spinal cord of lampreys controlling various modes of swimming behavior (Grillner et al., 1995; Grillner, 1997; Viana Di Prisco et al., 1997) and that in the mammalian brainstem controlling different forms of breathing (Feldman and Smith, 1989; Ramirez and Richter, 1996), separation of synaptic modulation of network properties from synaptic modulation of cellular properties is a difficult task, owing to the complex interactions between the cellular elements composing the central pattern generators. In Xenopus larvae, two distinct motor patterns, swimming and struggling, are driven by a common premotor pathway (Soffe, 1993). They can be controlled by the level of excitation within the spinal motor circuitry and need not involve the activity of a specific external neuromodulator (Soffe, 1996). In invertebrates, on the other hand, modulation of premotor control circuits appears to be far more distributed, and no clear behavior-specific segregation at the level of individual target cells has been described (Harris-Warrick and Marder, 1991; Marder and Calabrese, 1996).

Preliminary evidence from light-microscopical studies, so far lacking direct physiological confirmation, indicates that the segregation of behavior-specific inputs onto different cell types might be a general feature in pacemaker nuclei of various gymnotiform fish. Although Sternopygus, for instance, does not perform a JAR (Bullock, 1969) or produce chirps (Hopkins, 1974a), it can interrupt its EOD for several seconds via NMDA receptor-mediated input originating from the SPPn and presumably terminating on relay cells. In addition, Sternopygus can gradually raise its EOD frequency via NMDA receptor-mediated input originating from 


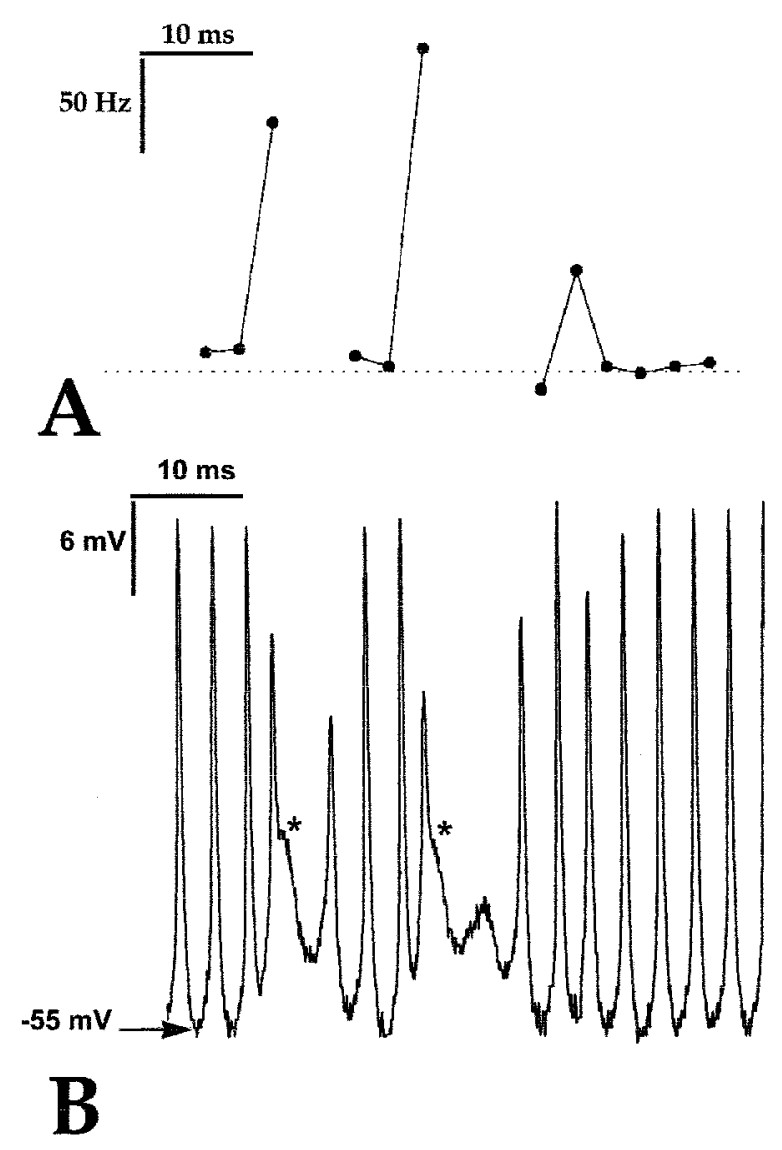

Figure 5. During interruptions of an otherwise stable spike frequency $(A)$, intracellular recordings from a relay cell demonstrate unique membrane potential changes $(B)$. A plot of instantaneous spike frequency versus time $(A)$ illustrates the magnitude of frequency shifts before interruptions and rapid recovery to normal rhythm after interruptions. While recording intracellularly from a relay cell $(B)$, the appearance of a shoulder on the repolarizing phase of an action potential (asterisks) correlates with the occurrence of a brief interruption, i.e., a skipped spike. Resting frequency (frequency before interruption) was $351 \mathrm{~Hz}$. This interruption in the spike pattern of the cells was also relayed to the EOD. It was, however, never observed in pacemaker cells.

the diencephalic prepacemaker nucleus, which seems to project to pacemaker cells (Keller et al., 1991). Other possible examples for this segregation of synaptic input from different behavior-specific premotor pathways are found in Apteronotus (Heiligenberg et al., 1996) and Hypopomus (Kennedy and Heiligenberg, 1994; Spiro, 1997).

Maintaining segregation of premotor inputs to not only different target cells, but also to different receptor subtypes (Fig. 1), bears the potential of increasing the behavioral repertoire even more; the channel kinetics of AMPA-type receptors make them particularly well suited for brief and rapid accelerations, such as those occurring in most chirps, whereas NMDA-type receptors are better suited for long, gradual frequency increases. (Of course, this does not exclude the possibility that the slower, gradual EOD frequency changes observed during PPnG stimulation could also be caused by activity changes of PPnG cells occurring with a slow time constant.) Segregation of these receptors onto different cell types in the pacemaker nucleus would ensure reproducibility of each form of frequency modulation. Brief changes would be encoded more reliably by the output cell type, relay cells, because any internal noise would be least detri- mental to the generation of distinct, abrupt changes in firing rate at this peripheral level. On the other hand, stable sustained changes are more noise tolerant and can be introduced into the pacemaker network more "upstream" by the rhythm-generating cell type, pacemaker cells.

If separate excitatory inputs that carry different information streams, such as those generating chirps and those mediating EOD deceleration during the JAR, terminate onto the same cells within a premotor command nucleus, in our example onto relay cells (Fig. 1), they often involve different receptor subtypes. Similarly, in the oscine song system, inputs from two neostriatal nuclei, the lateral magnocellular nucleus of the neostriatum (IMAN) and high vocal center $(\mathrm{HVc})$, believed to carry auditory feedback and temporal patterning, respectively, terminate on neurons of the vocal premotor command nucleus, the robust nucleus of the archistriatum (Doupe and Konishi, 1991). However, the IMAN input is mediated by NMDA receptors, whereas the HVc input is mediated by AMPA receptors (Mooney, 1992; Mooney and Konishi, 1991). In the spinal locomotor circuit in lamprey, computer simulations have revealed that the relative contribution of NMDA and non-NMDA receptor-mediated inputs plays a significant role in modifying the frequency of rhythmic bursting in spinal motoneurons (Grillner et al., 1995). Thus, different swim rates can be attributed to differential weighting of NMDA-AMPA receptor-mediated inputs. When glutamatergic inputs from reticulospinal cells is weighted in favor of NMDA receptors, slow rates of rhythmic bursting emerge from the network. In contrast, higher rates of rhythmic bursting can be generated by weighing the glutamatergic inputs in favor of AMPA receptors. The cellular basis for this differential weighing of NMDA-AMPA receptor activation has, however, not yet been identified.

In the present study, the large conductance increases in relay cells lasting 8-20 msec during PPnC stimulation share remarkable similarity with measurements of purely AMPA receptormediated currents in rat retinal ganglion cells (Taschenberger et al., 1995). Because the time course of input resistance changes is expected to parallel the time course of postsynaptic currents, our results provide additional physiological evidence suggesting the involvement of AMPA receptor-mediated currents in relay cells underlying chirp-like communication behavior. Whether receptor desensitization and/or receptor deactivation shapes the time course of conductance changes in our system will be addressed in future studies. Arguably, the consistency of the behavior is dependent on rapid termination of postsynaptic currents. Prolonged depolarization of relay cells for $>20 \mathrm{msec}$ would be expected to additionally recruit NMDA receptors if Glu had not been sufficiently cleared from the synaptic cleft. This would, of course, shape the magnitude and time course of frequency modulations. An entirely different behavior could thus be sculpted at the subcellular level. Long EOD interruptions, which often occur during courtship (Hopkins, 1974b; Hagedorn and Heiligenberg, $1985)$, are characteristically three to five times longer in duration $(\geq 70 \mathrm{msec})$ than the short chirps investigated in the present study, which naturally occur during agonistic encounters (Hopkins, 1974b). It is thus tempting to speculate that the longer chirps in Eigenmannia could be attributed to a combined role for AMPA and NMDA receptors. Interestingly, long chirps can be elicited in Apteronotus, a related gymnotiform (Alves-Gomez et al., 1995), by electrical stimulation of cells in the SPPn. In contrast to Eigenmannia, this form of stimulation "turns on" an otherwise quiescent glutamatergic input to relay cells, which appears to be 


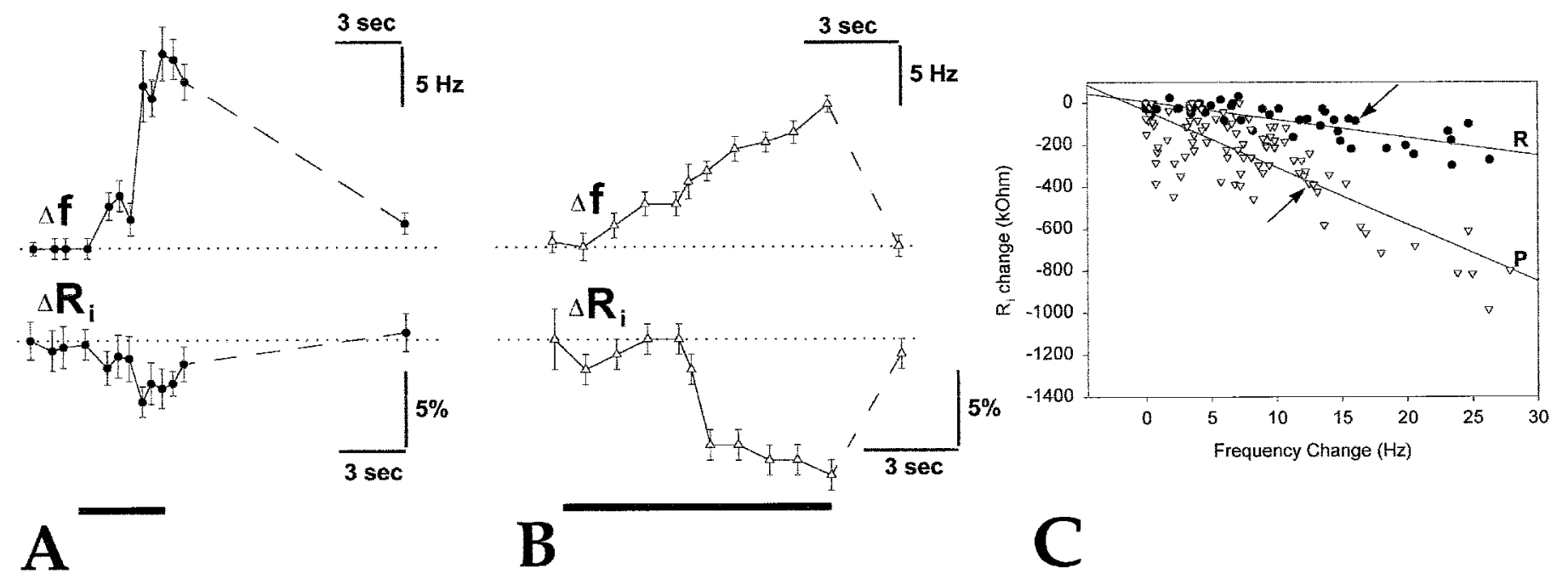

Figure 6. Time course of changes in spike frequency $(\Delta f$, top traces $)$ and input resistance $\left(\Delta R_{\mathrm{i}}\right.$, lower traces $)$ measured during PPnG stimulation in a relay cell $(A)$ and a pacemaker cell $(B)$. The duration of the stimulations is indicated by the thick horizontal bars. The input resistance of the relay cell $(A)$ decreased significantly less than that of the pacemaker cell $(B)$ during similar frequency changes. Individual data points are presented as an average \pm SEM of the amplitude of 10 downward voltage deflections. Respective spike frequencies before gradual frequency acceleration were $489(A)$ and 496 $\mathrm{Hz}(B)$. Input resistance before frequency acceleration was $2.6(A)$ and $3.4 \mathrm{M} \Omega(B)$. $C$, Changes in input resistance as a function of frequency changes during PPnG stimulation for the entire data sample of relay cells ( filled circles; 54 observations in four somata) and pacemaker cells (open triangles; 95 observations in four somata). The slopes of the regression lines were $-8.6 \mathrm{k} \Omega / \mathrm{Hz}$ for relay cells $(R)$ and $-27.1 \mathrm{k} \Omega / \mathrm{Hz}$ for pacemaker cells $(P)$. Regression coefficients differed significantly $(p<0.001)$. Arrows indicate the examples depicted in Figure $6 A, B$.
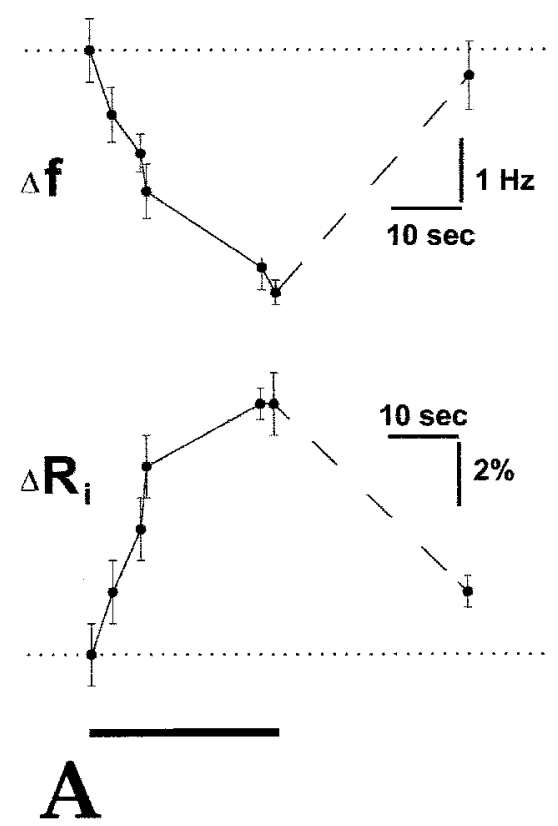
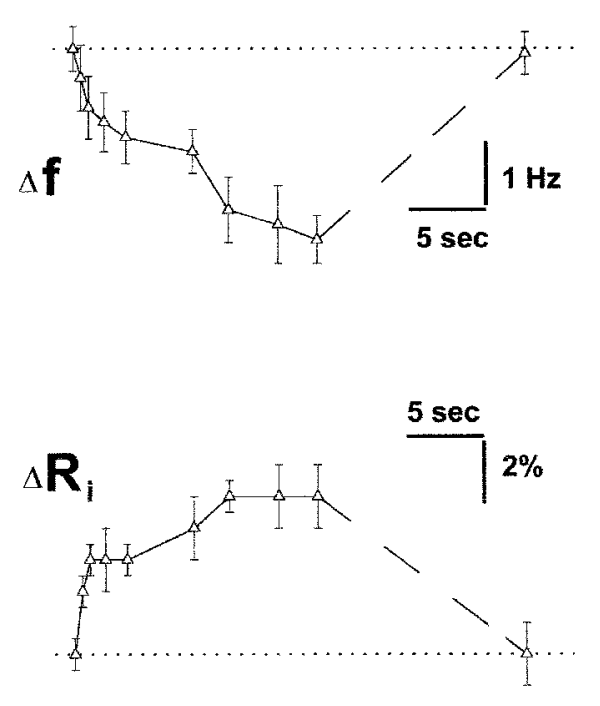

B

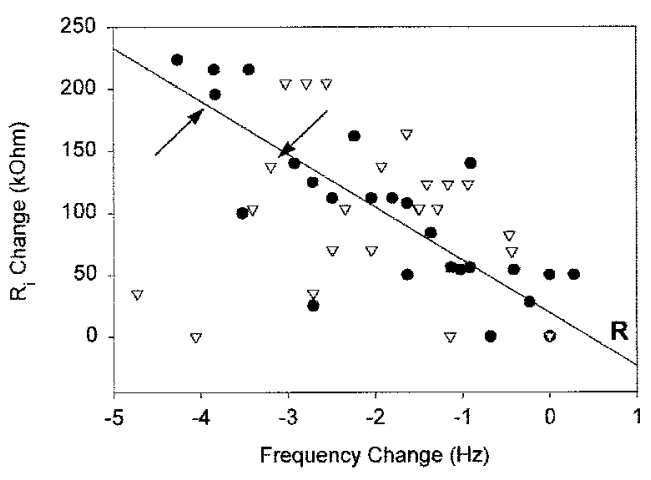

C

Figure 7. Time course of changes in spike frequency $(\Delta f$, top traces $)$ and input resistance $\left(\Delta R_{\mathrm{i}}\right.$, lower traces $)$ measured during $\mathrm{nE} \downarrow$ stimulation in a relay cell $(A)$ and a pacemaker cell $(B)$. The duration of the stimulations is indicated by the thick horizontal bars. The input resistance of the relay cell $(A)$ increased more than that of the pacemaker cell $(B)$ during similar frequency changes. Individual data points are presented as an average \pm SEM of the amplitude of 10 downward voltage deflections. Respective spike frequencies before frequency deceleration were $337(A)$ and $360 \mathrm{~Hz}(B)$. Input resistance before frequency deceleration was $2.7(A)$ and $4.1 \mathrm{M} \Omega(B)$. C Changes in input resistance as a function of frequency changes during nE $\downarrow$ stimulation for the entire data sample of relay cells ( filled circles; 29 observations in four somata) and pacemaker cells (open triangles; 33 observations in four somata). The slope of the regression line was $-42.8 \mathrm{k} \Omega / \mathrm{Hz}(R)$. In pacemaker cells, this relationship could not be fitted with a linear function $\left(r^{2}=0.07\right)$; therefore, the linear regression line was omitted for pacemaker cells.

mediated by NMDA receptors (Heiligenberg et al., 1996). The behavioral result is a long chirp that resembles those produced by males during courtship.

Especially during such longer chirps resulting in interruptions of the otherwise continuous spike train (and EOD), a shoulder- like phenomenon during prolonged spike duration was commonly observed in relay cell recordings (Fig. 5). This shares some essential features with well described modulations of intrinsic membrane properties found in other systems, such as the spinal locomotor network in lamprey and the mammalian respiratory 


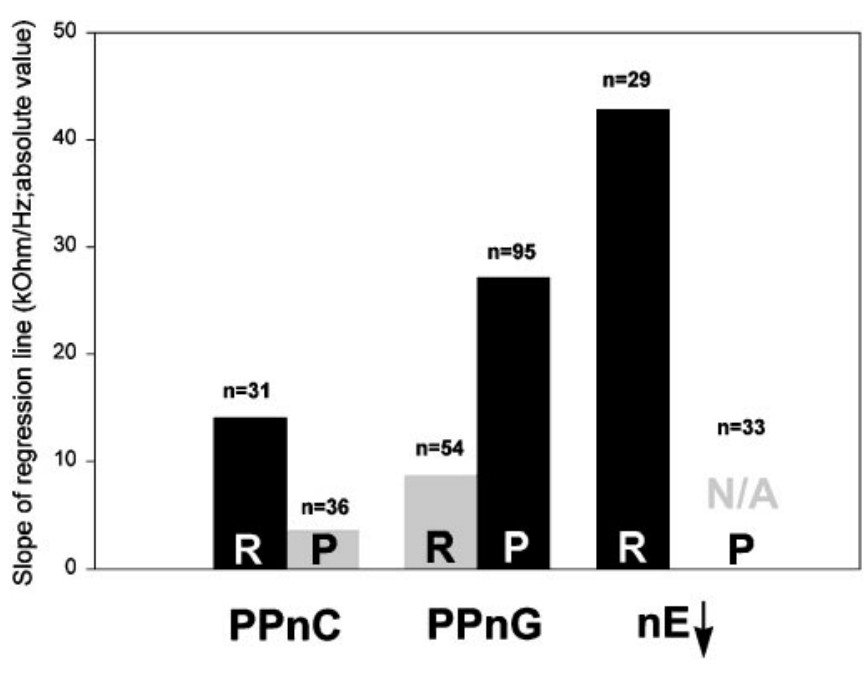

Target cell type Coupled cell type

Figure 8. Slope of regression lines compared between cell types and across stimulation sites. The input resistance of relay cells was more affected than that of pacemaker cells during both PPnC and $\mathrm{nE} \downarrow$ stimulation. In contrast to the decrease in input resistance observed during PPnC stimulation, the input resistance during $\mathrm{nE} \downarrow$ stimulation increased. The decrease in input resistance observed in pacemaker cells during PPnG stimulation was significantly different from the decrease in input resistance in relay cells during PPnC stimulation. $R$, Relay cells; $P$, pacemaker cells; $n$, number of data points used to calculate each regression line; $N / A$, not available.

network in the medulla (Feldman and Smith, 1989; Grillner et al., 1995; Ramirez and Richter, 1996). Recent pharmacological experiments in the isolated pacemaker nucleus of Apteronotus might help to explain this phenomenon. These studies have indicated a role for a nickel- and cadmium- sensitive $\mathrm{Ca}^{2+}$ current in maintaining the normal resting frequency of spike discharge (Smith and Zakon, 1997). Because voltage-gated $\mathrm{Ca}^{2+}$ channel blockers had no effect on the ongoing spike rate, the type of $\mathrm{Ca}^{2+}$ channel contributing to the resting oscillation frequency has yet to be more fully characterized (Smith and Zakon, 1997). As reported in other oscillatory systems (Grillner et al., 1995; Ramirez and Richter, 1996; Grillner, 1997), modulation of $\mathrm{Ca}^{2+}$-activated channels is an especially effective means to alter spike rates. It is tempting to speculate that the spike broadening we observed could be caused by an increased $\mathrm{Ca}^{2+}$ influx during membrane depolarization. It appears therefore that different cellular mechanisms might have contributed to the widening of the behavioral repertoire of weakly electric fish.

Results from recent lesion experiments in the electrosensory system of gymnotiform fish suggest that the sensory signals evoking chirp-like communication behavior and yielding a JAR are processed in segregated pathways as well (Metzner and Juranek, 1997b). The first order nucleus of the electrosensory system, the electrosensory lateral line lobe, consists of four segments, three of them receiving identical input from electroreceptors. Although the centromedial segment was both necessary and sufficient for the JAR, it did not affect the communicative response to external electric signals. Conversely, the lateral segment did not affect the JAR but was necessary and sufficient to evoke communication behavior. Combined with the findings described in the present study, this system illustrates that sensorimotor control of different behaviors can occur in strictly segregated channels from the sensory input of the brain all through to its motor output.

What is the reason for the distinctly distributed organization of the electrosensory and premotor systems in these fish? For the existence of multiple electrosensory maps, it is conceivable that duplication of existing brain maps could efficiently accommodate the increased information flow associated with a growth in the behavioral repertoire, as originally proposed for the sensory maps in mammalian cortex (Allman and Kaas, 1971; Kaas, 1982; see also Metzner and Juranek, 1997b). On the premotor side, accurate electrolocation requires an extremely low jitter in EOD discharges (Bullock, 1969; Heiligenberg, 1991; Moortgat et al., 1998). This demand for highly synchronous and regular discharge of the pacemaker nucleus probably was the leading selective pressure yielding the strict functional separation of its cellular components into pacemaking units, i.e., pacemaker cells, and output units, i.e., relay cells (Bennett et al., 1967). This rigid design of the premotor command nucleus may, in turn, have required its synaptic inputs to remain distinctly segregated to produce the different motor patterns.

\section{REFERENCES}

Allman JM, Kaas JH (1971) A representation of the visual field in the caudal third of the middle temporal gyrus of the owl monkey (Aotus trivirgatus). Brain Res 31:85-105.

Alves-Gomez JA, Orti G, Haygood M, Heiligenberg W, Meyer A (1995) Phylogenetic analysis of the South American electric fishes (order Gymnotiformes) and the evolution of their electrogenic system: a synthesis based on morphology, electrophysiology, and mitochondrial sequence data. Mol Biol Evol 12:298-318.

Arshovsky YI, Orlovsky GN, Panchin YV, Roberts A, Soffe SR (1993) Neuronal control of swimming locomotion: analysis of the pteropod mollusc Clione and embryos of the amphibian Xenopus. Trends Neurosci 16:227-233.

Bennett MV (1997) Gap junctions as electrical synapses. J Neurocytol 26:349-366.

Bennett MV, Barrio LC, Bargiello TA, Spray DC, Hertzberg E, Saez JC (1990) Gap junctions: new tools, new answers, new questions. Neuron 6:305-320.

Bennett MVL (1971) Electric organs. In: Fish physiology (Hoar RS, Randall DJ, eds), pp 347-491. New York: Academic.

Bennett MVL, Pappas GD, Gimenez M, Nakajima Y (1967) Physiology and ultrastructure of electrotonic junctions. IV. Medullary electromotor nuclei in gymnotid fish. J Neurophysiol 30:236-300.

Blitz DM, Nusbaum MP (1997) Motor pattern selection via inhibition of parallel pathways. J Neurosci 17:4965-4975.

Brodfuehrer PD, Burns A (1995) Neuronal factors influencing the decision to swim in the medicinal leech. Neurobiol Learn Mem 63:192-199.

Bullock TH (1969) Species differences in effect of electroreceptor input on electric organ pacemakers and other aspects of behavior in electric fish. Brain Behav Evol 2:85-118.

Bullock TH, Hamstra RH, Scheich H (1972) The jamming avoidance response of high frequency electric fish. I. General features. J Comp Physiol [A] 77:1-22.

Doupe AJ, Konishi M (1991) Song-selective auditory circuits in the vocal control system of the zebra finch. Proc Natl Acad Sci USA 88:11339-11343.

Dye J, (1991) Ionic and synaptic mechanisms underlying a brainstem oscillator: an in vitro study of the pacemaker nucleus of Apteronotus. J Comp Physiol [A]168:521-532.

Dye J, Heiligenberg W, Keller CH, Kawasaki M (1989) Different classes of glutamate receptors mediate distinct behaviors in a single brainstem nucleus. Proc Natl Acad Sci USA 86:8993-8997.

Elekes K, Szabo T (1982) Synaptic organization in the pacemaker nucleus of a medium-frequency weakly electric fish, Eigenmannia sp. Brain Res 237:267-281.

Elekes K, Szabo T (1985) Synaptology of the medullary command (pacemaker) nucleus of the weakly electric fish (Apteronotus leptorhynchus) with particular reference to comparative aspects. Exp Brain Res 60:509-520. 
Feldman JL, Smith JC (1989) Cellular mechanisms underlying modulation of breathing pattern in mammals. Ann NY Acad Sci 563:114-130. Grillner S (1997) Ion channels and locomotion. Science 278:1087-1088. Grillner S, Deliagin T, Ekeberg O, El Manira A, Hill RH, Lansner A, Orlovsky GN, Wallen P (1995) Neural networks that co-ordinate locomotion and body orientation in lamprey. Trends Neurosci 18:270-279.

Hagedorn M, Heiligenberg W (1985) Court and spark: electric signals in the courtship and mating of gymnotoid fish. Anim Behav 33:254-265.

Harris-Warrick RM, Marder E (1991) Modulation of neural networks for behavior. Annu Rev Neurosci 14:39-58.

Heiligenberg W (1991) Neural nets in electric fish. Cambridge, MA: MIT.

Heiligenberg W, Metzner W, Wong CJH, Keller CH (1996) Motor control of the jamming avoidance response of Apteronotus leptorhynchus: evolutionary changes of a behavior and its neuronal substrates. J Comp Physiol [A] 179:653-674.

Hopkins CD (1974a) Electric communication in the reproductive behavior of Sternopygus macrurus Gymnotoidei. Z Tierpsychol 35:518-535.

Hopkins CD (1974b) Electric communication: functions in the social behavior of Eigenmannia virescens. Behaviour 50:268-270.

Juranek J, Metzner W (1997) Cellular characterization of synaptic modulations of a neuronal oscillator in electric fish. J Comp Physiol [A] 181:393-414

Kaas JH (1982) The segregation of function in the nervous system: why do sensory systems have so many subdivisions? Contrib Sens Physiol 7:201-240.

Katz PS (1995) Intrinsic and extrinsic neuromodulation of motor circuits. Curr Opin Neurobiol 5:799-808.

Kawasaki M, Heiligenberg W (1989) Distinct mechanisms of modulation of a neuronal oscillator in electric fish Hypopomus. J Comp Physiol [A] 165:731-741.

Kawasaki M, Maler L, Rose G (1988) Anatomical and functional organization of the prepacemaker nucleus in gymnotiform electric fish: the accommodation of two behaviors in one nucleus. J Comp Neurol 276:113-131.

Keller CH, Heiligenberg W (1989) From distributed sensory processing to discrete motor representations in the diencephalon of the electric fish, Eigenmannia. J Comp Physiol [A] 164:565-576.

Keller M, Maler L, Heiligenberg W (1990) Structural and functional organization of a diencephalic sensory-motor interface in the gymnotiform fish, Eigenmannia. J Comp Physiol [A] 169:441-450.

Keller CH, Kawasaki M, Heiligenberg W (1991) The control of pacemaker modulation for social communication in the weakly electric fish Sternopygus. J Comp Physiol [A] 169:441-450.

Kennedy G, Heiligenberg W (1994) Ultrastructural evidence of GABAergic inhibition and glutamatergic excitation in the pacemaker nucleus of the gymnotiform electric fish, Hypopomus. J Comp Physiol [A] $174: 267-280$.
Marder E, Calabrese RL (1996) Principles of rhythmic motor pattern generation. Physiol Rev 76:687-717.

Metzner W (1993) The jamming avoidance response in Eigenmannia is controlled by two separate motor pathways. J Neurosci 13:1862-1878.

Metzner W, Juranek J (1997a) A method to biotinylate and histochemically visualize ibotenic acid for pharmacological inactivation studies. J Neurosci Methods 76:143-150.

Metzner W, Juranek J (1997b) A sensory brain map for each behavior? Proc Natl Acad Sci USA 94:14798-14803.

Metzner W, Viete S (1996a) The neuronal basis of communication and orientation in the weakly electric fish, Eigenmannia. I. Communication behavior or seeking a conspecific's response. Naturwissenschaften 83:6-14.

Metzner W, Viete S (1996b) The neuronal basis of communication and orientation in the weakly electric fish, Eigenmannia. II. Electrolocation and avoidance of jamming by neighboring conspecifics. Naturwissenschaften 83:71-77.

Mooney R (1992) Synaptic basis for developmental plasticity in a birdsong nucleus. J Neurosci 12:2464-2477.

Mooney R, Konishi M (1991) Two distinct inputs to an avian song nucleus activate different glutamate receptor subtypes on individual neurons. Proc Natl Acad Sci USA 88:4075-4079.

Moortgat KT, Keller CH, Bullock TH, Sejnowski TJ (1998) Submicrosecond pacemaker precision is behaviorally modulated: the gymnotiform electromotor pathway. Proc Natl Acad Sci USA 95:4684-4689.

Ramirez J-N, Richter DW (1996) The neuronal mechanisms of respiratory rhythm generation. Curr Opin Neurobiol 6:817-825.

Smith TG, Zakon H (1997) Potassium and calcium currents regulate firing frequency of pacemaker nucleus neurons in Apteronotus leptorhynchus. Soc Neurosci Abstr 27:101.

Soffe SR (1993) Two distinct rhythmic motor patterns are driven by common premotor and motor neurons in a simple vertebrate spinal cord. J Neurosci 13:4456-4469.

Soffe SR (1996) Motor patterns for two distinct rhythmic behaviors evoked by excitatory amino acid agonists in the Xenopus embryo spinal cord. J Neurophysiol 75:1815-1825.

Spiro J (1997) Differential activation of glutamate receptor subtypes on a single class of cells enables a neural oscillator to produce distinct behaviors. J Neurophysiol 78:835-847.

Spiro JE, Brose N, Heinemann SF, Heiligenberg W (1994) Immunolocalization of NMDA receptors in the central nervous system of weakly electric fish: functional implications for the modulation of a neuronal oscillator. J Neurosci 14:6289-6299.

Taschenberger H, Engert F, Grantyn R (1995) Synaptic current kinetics in a solely AMPA-receptor-operated glutamatergic synapse formed by rat retinal ganglion neurons. J Neurophysiol 74:1123-1136.

Viana Di Prisco G, Pearlstein E, Robitaille R, Dubuc R (1997) Role of sensory-evoked NMDA plateau potentials in the initiation of locomotion. Science 278:1122-1125. 